\title{
MRI-guided stereotactic radiation therapy for prostate cancer: a mini review.
}

\author{
Aryavarta M S Kumar* \\ University Hospitals, Seidman Cancer Center, Cleveland OH, USA
}

Keywords: Prostate cancer, Cancer diagnoses, Partial gland therapy, Prostate malignancy, Photodynamic therapy. Accepted on 24 November, 2017

\section{Mini Review}

The incidence of prostate cancer remains very high and is the second leading cause of cancer related death in the USA $[1,2]$. Worldwide prostate cancer accounts for $15 \%$ of all male cancer diagnoses. Important within this large group of patients is identifying the different risk groups (low risk, intermediate risk, high risk) and subgroups within them. While treatment advances involving surgery, radiation, hormonal therapy, and systemic treatments continue to provide improved outcomes in more aggressive prostate cancer, there have also been new paradigms describing treatment de-escalation for lower risk prostate cancer. Some of this involves active surveillance protocols with no definitive treatment [3].

While active surveillance may be appropriate for some patients, there appears to be a role for a partial prostate treatment in some low risk patients $[4,5]$. Dose escalation has been shown to improve biochemical outcomes from several studies, but this comes at a cost to the adjacent organs that also receive a higher dose [6,7]. Radiation proctitis and cystitis while less common are likely underreported and can cause long term morbidity for patients [8]. Partial gland therapy would aim to deliver a high therapeutic dose to specifically identified targets within the prostate gland and in most cases spare the higher doses of radiation from reaching the rectum and bladder [5, 9]. Important in this paradigm is being able to identify areas of disease $[9,10]$. For example, current biopsy sample the prostate via the peripheral zone however, may be underestimating anterior gland disease. Additionally, small areas could theoretically be missed.

Improvements in imaging has led to renewed interest in using dynamic contrast enhanced (DCE) MRI to evaluate for prostate malignancy [11]. In fact a PI-RADS system has been created to help grade the radiographic features [12]. However, evaluation by imaging alone currently does not yield a high sensitivity or specificity $[9,10]$. A biopsy confirmation of these lesions is important to evaluate the true and false positive results. True positives may then be candidates for partial gland therapy on protocol. In addition, it is also possible for a prostate cancer to not be readily visualized using DCE-MRI. It should be stated that partial gland therapy is not currently standard of care and thus practitioners are encouraged to study this on protocol at this time. Focal therapy has been attempted in some published studies. In fact, focused ultrasound, thermotherapy, brachytherapy, photodynamic therapy and stereotactic radiosurgery have been attempted $[4,5]$.
While standard external beam radiation may take up to 8-9 weeks to complete the delivery of a definitive dose to the prostate for prostate cancer, more recent advances in radiation have allowed the safe delivery of higher doses per fraction. Stereotactic radiation has introduced the concept of treating a prostate cancer in a few fractions completed in 1-2 weeks. The exact dose and fractionation are still under investigation however, radiation doses similar to current brachytherapy schedules appear to be the most promising. Outcomes appear comparable to standard radiation schedules [13].

One advantage to stereotactic radiation is the excellent dosimetry and the non-invasive nature of the process. A stereotactic course could be completed in a matter of 5 days for example in an every other day schedule (total elapsed days within 2 weeks). When combined with partial gland therapy, the therapeutic index can be very favourable as the higher doses are likely not to reach all the important adjacent normal organs. On a larger scale, focal therapy represents a paradigm change to more customized treatment plans for patients with prostate cancer. Investigations are on-going at this time, but partial gland SBRT guided by DCE MRI remains a promising therapeutic option for select prostate cancer patients.

\section{References}

1. Global Cancer Factrs and Figures.

2. Klotz L, Zhang L, Lam A, et al. Clinical results of longterm follow-up of a large, active surveillance cohort with localized prostate cancer. J Clin Oncol. 2010;28(1):126-31.

3. Valerio M, Ahmed HU, Emberton M, et al. The role of focal therapy in the management of localised prostate cancer: a systematic review. Eur Urol. 2014;66(4):732-51.

4. Eggener SE, Scardino PT, Carroll PR, et al. Focal therapy for localized prostate cancer: a critical appraisal of rationale and modalities. J Urol. 2007;178(6):2260-7.

5. Bolzicco G, Favretto MS, Scremin E, et al. Image-guided stereotactic body radiation therapy for clinically localized prostate cancer: preliminary clinical results. Technol Cancer Res Treat. 2010;9(5):473-7.

6. Friedland JL, Freeman DE, Masterson-Mcgary ME, et al. Stereotactic body radiotherapy: an emerging treatment approach for localized prostate cancer. Technol Cancer Res Treat. 2009;8(5):387-92.

7. Zelefsky MJ, Chan H, Hunt M, et al. Long-Term Outcome of High Dose Intensity Modulated Radiation Therapy for Patients With Clinically Localized Prostate Cancer. The Journal of Urology. 2006;176(4):1415-9. 
Citation: M S Kumar, Aryavarta. MRI-guided stereotactic radiation therapy for prostate cancer: a mini review. J Cancer Immunol Ther. 2017;1(1):9-11.

8. Ellis RJ, Kaminsky DA, Zhou EH, et al. Ten-year outcomes: the clinical utility of single photon emission computed tomography/computed tomography capromab pendetide (Prostascint) in a cohort diagnosed with localized prostate cancer. Int J Radiat Oncol Biol Phys. 2011;81(1):29-34.

9. Pinto PA, Chung PH, Rastinehad AR, et al. Magnetic resonance imaging/ultrasound fusion guided prostate biopsy improves cancer detection following transrectal ultrasound biopsy and correlates with multiparametric magnetic resonance imaging. J Urol. 2011;186(4):1281-5.

10. Kirkham APS, Emberton M, Allen C. How Good is MRI at Detecting and Characterising Cancer within the Prostate? European Urology. 2006;50(6):1163-75.
11. Weinreb JC, Barentsz JO, Choyke PL, et al. PI-RADS Prostate Imaging-Reporting and Data System. European Urology. 2016;69(1):16-40.

12. King CR, Freeman D, Kaplan I, et al. Stereotactic body radiotherapy for localized prostate cancer: Pooled analysis from a multi-institutional consortium of prospective phase II trials. Radiotherapy and Oncology. 2013;109(2):217-21.

\section{*Correspondence to}

Aryavarta M S Kumar

University Hospitals, Seidman Cancer Center, USA

E-mail: aryavarta.kumar@uhhospitals.org 